\title{
ANISOTROPY IN SOME SOFT MAGNETIC MATERIALS
}

\author{
W. B. HUTCHINSON and J. G. SWIFT \\ Department of Physical Metallurgy, University of Birmingham, Edgbaston, \\ Birmingham 15, England; and West Midland Gas Board, Solihull, England
}

(Received March 20, 1972, in final form June 9, 1972)

\begin{abstract}
Orientation distribution data are presented for low carbon steel and silicon iron after different rolling and annealing schedules. Magnetic properties which depend solely on orientation can be well described using such distributions. A general analysis is demonstrated by which other properties may be divided into two components which are respectively dependent and independent of orientation. An example of this, a.c. power loss, is considered in detail. It is shown that the orientation-dependence of power loss derives entirely from the hysteresis component. The classical eddy current loss together with an 'anomalous' loss of similar magnitude consţitute the orientationindependent part. The analysis may be used to predict properties under a range of conditions in textured materials.
\end{abstract}

\section{INTRODUCTION}

A very important commercial application of texture in metals lies in materials for electrical transformer cores and other electro-magnetic operations. For many years, the standard material for core laminations of power transformers has been $3 \frac{1}{4} \%$ silicon steel treated to exhibit a very strong (110)[001] orientation, or Goss texture. ${ }^{1}$ This grain oriented structure is produced by secondary recrystallisation and has a grain size in the range 3 to $10 \mathrm{~mm}$ in the plane of the sheet, which is typically $0.3 \mathrm{~mm}$ thick. The good magnetic properties of this material are largely due to the fact that the easy directions of magnetisation $\langle 001\rangle$ in the different grains are aligned along a single direction in the sheet.

Power losses during alternating magnetisation of transformer cores have several origins. There is the classical eddy current loss $^{2}$ which depends most strongly on resistivity and sheet thickness, and is essentially independent of orientation. Conversely, the static hysteresis loss is very strongly dependent on the direction of magnetisation. Finally, there is the so called anomalous loss for which several explanations have been suggested. Micro eddy currents and damping effects at moving domain boundaries have been proposed by Pry and Bean ${ }^{3}$ and $\mathrm{Lee}^{4}$ as origins of the anomaly. However, Overshott et al. ${ }^{5}$ also consider that domain boundary bowing at the free surfaces of thin sheet is an additional contribution. Despite the importance of preferred orientation to the magnetic properties, there has been rather little direct evidence published concerning the relationships. McCarty et al. ${ }^{6}$ found that permeability and power loss were most strongly affected by rotational deviations from the ideal (110)[001] orientation around the sheet plane normal. Misorientations around axes perpendicular to this were not found to influence the properties strongly. However, a contrary observation was made by Daniels ${ }^{7}$ who found that large rotational deviations around the transverse direction caused high power losses. It may be remarked that rotations around the normal and transverse directions are crystallographically identical but, because of the two dimensional nature of the grain structure, these may cause different magnetic closure domain structures. From a practical viewpoint, the Goss textured silicon steel is awkward to examine for preferred orientation. The grain size is too large for pole figure determination by $x$-ray analysis, and grain by grain measurements using Laue or etch pit methods are extremely time consuming.

Generally speaking, one may divide magnetic properties into those which are dependent on both orientation and microstructure (e.g. power loss), and those which depend solely on orientation. Examples of the latter type are the magnetostatic energy at saturation and the magnetic torque, which is the differential of that energy with respect to angular displacement. The torque magnetometer has been widely used for testing preferred orientation of electrical steels for quality control. Analysis of torque curves is normally based on the equation for the magnetostatic energy $E^{8}$

$$
E=K_{0}+K_{1}\left(l^{2} m^{2}+m^{2} n^{2}+n^{2} l^{2}\right)+K_{2} l^{2} m^{2} n^{2} \ldots
$$


This is a rationalisation of symmetry considerations for a cubic crystal in which the magnetisation vector has the direction cosines $l, m, n$. The coefficients $K_{0}, K_{1}, K_{2}$ are termed magnetocrystalline anisotropy coefficients. Where shape anisotropy can be ignored, as for example in a thin disc whose plane includes the field direction, the differential of $E$ with respect to angle gives the torque experienced by the sample. This can be readily measured. A solution of this differential for the $K_{1}$ term of a general orientation has been given by Tarasov and Bitter ${ }^{9}$. Usually subsequent terms are of little weight. Particular solutions for high symmetry orientations can be found in most text books of magnetic materials.

In order to apply the above type of analysis to a polycrystalline metal, one requires to know a distribution of the orientations present. This is not directly measurable but may be calculated from pole figure data using methods developed by Williams ${ }^{10}$ or Bunge ${ }^{11}$ and Roe $^{12}$. Bunge ${ }^{13}$ has also shown mathematically how a distribution function of orientations may be applied to calculate the magnetic torque behaviour of rolled sheet material.

Since the use of orientation distributions is rather new, there is little complete data as yet published concerning texture in steels. Bunge ${ }^{14}$ and Hutchinson and Swift ${ }^{15}$ have given results for low carbon steel cold rolled $70 \%$. The rolling texture is seen to consist primarily of a fibre texture with $\langle 111\rangle$ parallel to the sheet normal and another with $\langle 110\rangle$ along the rolling direction. There is a peak in the latter at approximately the orientation (223)[110]. Heckler and Granzow ${ }^{16}$ have presented data at various reductions between $0 \%$ and $80 \%$. These authors observed the (100)[011] orientation to be strongly populated at low reductions, but this may have been due to its presence in the starting texture. With continuing deformation the $\langle 111\rangle$ and $\langle 110\rangle$ fibre textures develop similar orientation densities, except for components in the latter fibre within $15^{\circ}$ of (110)[1̄10] which are suppressed. After annealing at $700^{\circ} \mathrm{C}$, the $\langle 110\rangle$ fibre texture is observed to become much depleted. There is also general agreement that the Goss orientation (110) [001] appears on primary recrystallisation.

\section{EXPERIMENTAL}

The work reported here was carried out on a low carbon rimming steel and a $1.8 \%$ silicon iron, the latter being of the type used commercially as low grade electrical steel. The rimming steel was cold rolled $50 \%, 70 \%$, and $90 \%$ and a part of each was subsequently annealed at $700^{\circ} \mathrm{C}$ for one hour. Discs of $3 \mathrm{~cm}$ diameter were punched from these for torque magnetometer measurements. The silicon iron was first annealed for one hour at $1050^{\circ} \mathrm{C}$ and furnace cooled to form a coarse grain structure. This was followed by $60 \%$ cold reduction and a recrystallisation anneal of one hour at $700^{\circ} \mathrm{C}$. Duplicate test specimens of dimensions $3 \mathrm{~cm} \times$ $30 \mathrm{~cm}$ were cut with the long axes at angles of $0^{\circ}, 15^{\circ}, 30^{\circ}, 45^{\circ}, 60^{\circ}, 75^{\circ}$, and $90^{\circ}$ to the rolling direction. Finally, all the strip specimens were chemically polished to remove inhomogeneous surface layers and strained layers of the sheared edges.

Composite specimens of all the samples were made by soldering the sheets together and then $1.5 \mathrm{~cm}$ diameter spheres were machined from the composites. Complete (200) and (222) pole figures were obtained by the Jetter-Borie technique ${ }^{17}$ from these spherical specimens. The pole figure data were also used to calculate three dimensional orientation distribution plots using the biaxial pole figure program of Williams. ${ }^{10}$ This program gives solutions for the orientation density $(t)$ at 1296 different orientations. The definition of angles used by Williams is given in Figure 1. One may also note that for a sheet plane orientation defined by the angles $\rho$ and $\alpha$, any direction lying in the plane defined by an angle $\beta$ has direction cosines $l, m$, and $n$ which are given by the relationships

$$
\begin{gathered}
l=\sin \beta\left(1-\sin ^{2} \rho \sin ^{2} \alpha\right)^{\frac{1}{2}} \\
m=-\left(\cos \rho \cos \beta+\sin ^{2} \rho \sin \alpha \cos \alpha \sin \beta\right) / \\
\quad\left(1-\sin ^{2} \rho \sin ^{2} \alpha\right)^{\frac{1}{2}} \\
n=(\sin \rho \cos \alpha \cos \beta-\sin \rho \cos \rho \sin \alpha \sin \beta) / \\
\left(1-\sin ^{2} \rho \sin ^{2} \alpha\right)^{\frac{1}{2}}
\end{gathered}
$$

Hence, throughout the range of values of $\alpha, \rho$, and $\beta$ one can solve for the bracketed terms in Eq. (1).

Magnetic torque curves were obtained from the rimming steel samples using a conventional torsionwire magnetometer. Measured values were corrected according to the specimen dimensions to express them as torque per unit volume.

The strips of silicon iron were tested for total power loss at 30,50 , and $60 \mathrm{~Hz}$ with a maximum induction of 1.0 tesla. In addition, complete B-H loops were measured using a d.c. permeameter for $B_{\max }$ equal to 1.0 tesla. From these graphs the 


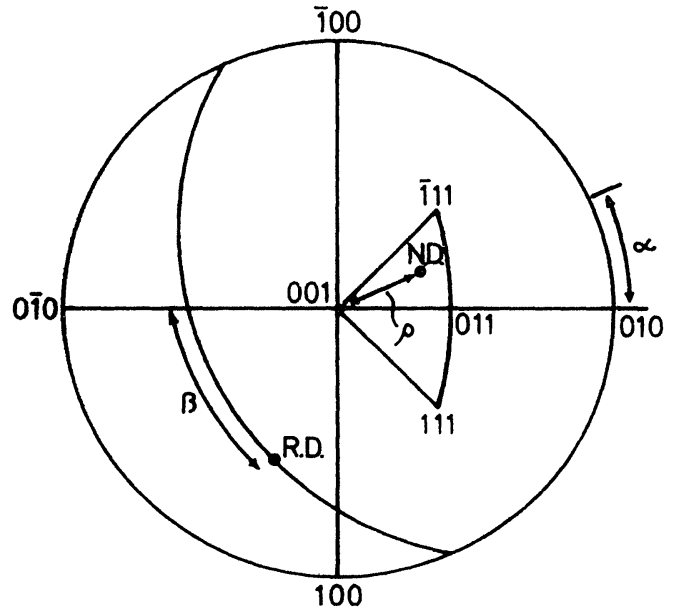

FIGURE 1 The definition of angular parameters.

remanence and coercive force were determined, and the hysteresis loss per cycle was calculated from the area of the loop.

\section{RESULTS AND DISCUSSION}

The orientation density plots for rimming steel cold rolled $50 \%$ and $90 \%$ are shown in Figures 2 and 3. That for the $70 \%$ reduction has been given previously. ${ }^{15}$ It is clear that the texture is already well developed at $50 \%$ and at this stage it differs little in form from that at $90 \%$. The main change which occurs with increasing reduction is strengthening of the major components at the expense of all other orientations. In particular, the density in orientations close to (223)[110] increases sharply and these become the strongest single components. The

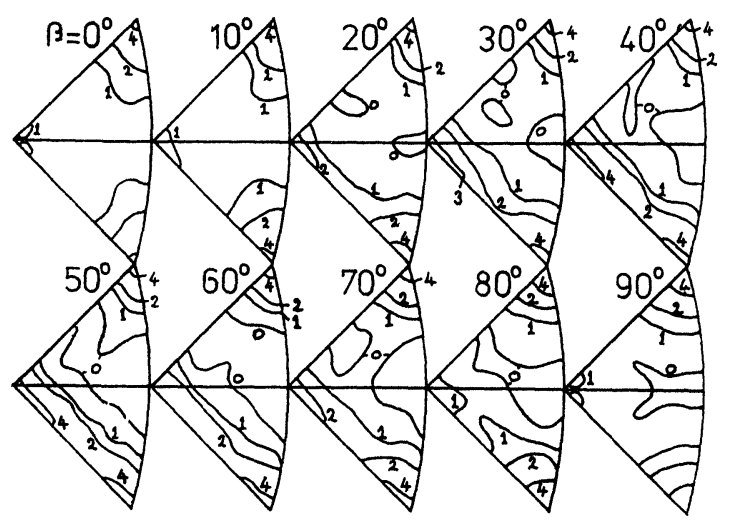

FIGURE 2 Biaxial pole figure for rimming steel rolled $50 \%$. orientation distributions after annealing at $700^{\circ} \mathrm{C}$ are shown in Figures 4 and 5. While the fibre texture with $\langle 111\rangle$ along the normal direction is little affected by the heat treatment, that with $\langle 110\rangle$ along the rolling direction is almost completely eliminated. The results of Bunge ${ }^{14}$ at $70 \%$ reduction indicate a similar behaviour although in that case the $\langle 111\rangle$ components were strengthened, while here they were observed to diminish somewhat. There is good qualitative agreement between the present observations and the theory of Dillamore et al. ${ }^{18}$ which is based on a sub-grain growth model for nucleation of recrystallisation. Analyses based on oriented growth assuming $30^{\circ}$ rotations around $\langle 110\rangle$ poles are not successful in predicting most of the ennealing texture components.

In addition to the $\langle 111\rangle$ orientations, there are two prominent annealing texture components. After low reduction the Goss component (110)[001] is very significant, while after heavy reduction there

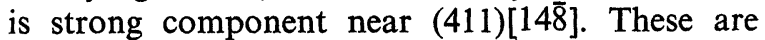
believed to derive from transition band and grain boundary nucleation sites respectively, and will be considered in detail elsewhere. ${ }^{19,20}$ The orientation distribution for the silicon iron (Figure 6) is similar to the rimming steel (Figure 4) but has a stronger Goss component because of the coarser initial grain size employed.

In order to compute torque magnetometer curves for the polycrystalline structures, values were first calculated for the 1296 single orientations which are used for plotting the biaxial pole figures. To do this $l, m$, and $n$ in Eq. (1) were expressed in terms of the angles $\alpha, \rho$, and $\beta$, and the expression was differentiated with respect to $\beta$. Values of $K_{1}$ and $K_{2}$ were assumed to be $420 \times 10^{3}$ and $150 \times 10^{3} \mathrm{erg} /$ $\mathrm{cm}^{3}$ respectively. ${ }^{21}$ An arithmetic mean of all the torque values was then taken after weighting the individual values by the orientation density factor $t$. A general texture component $\{h k l\}\langle u v w\rangle$ is represented in a sheet by four physically distinct orientations. For any single testing direction in the sheet plane, two of these behave identically and their properties are related to the other pair by reflection in the mirror planes of symmetry in the texture. Since the Williams representation, for economy of space, presents only one of the four components, a symmetry operation was performed to generate the properties of the missing variant.

Examples of the computed and measured torque curves are shown in Figures 7 and 8 for the $50 \%$ annealed and $90 \%$ rolled specimens. Results for the specimens after $70 \%$ reduction are given else- 


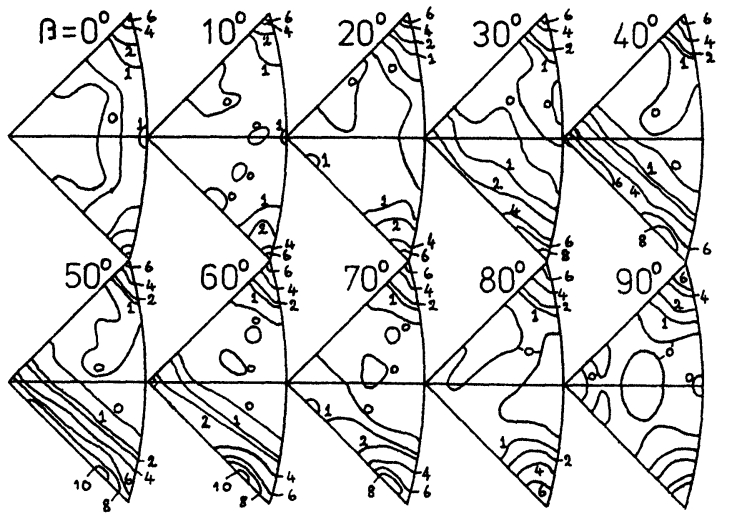

FIGURE 3 Biaxial pole figure for rimming. steel rolled $90 \%$.

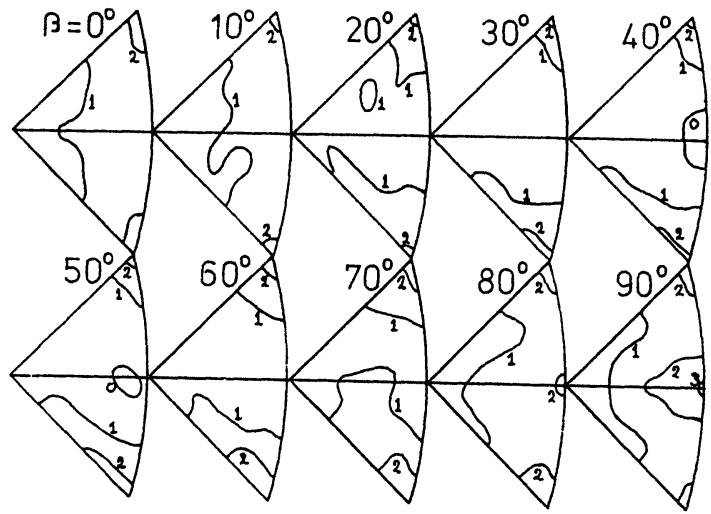

FIGURE 4 Biaxial pole figure for rimming steel rolled $50 \%$ and annealed at $700^{\circ} \mathrm{C}$.

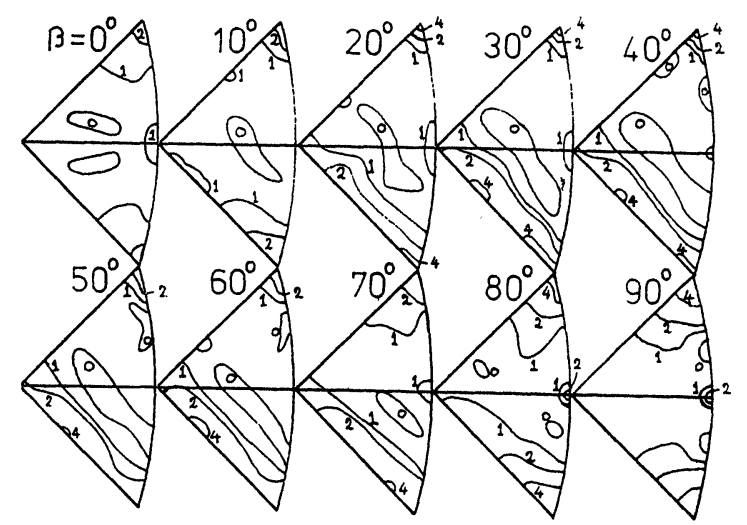

FIGURE 5 Biaxial pole figure for rimming steel rolled $90 \%$ and annealed at $700^{\circ} \mathrm{C}$.

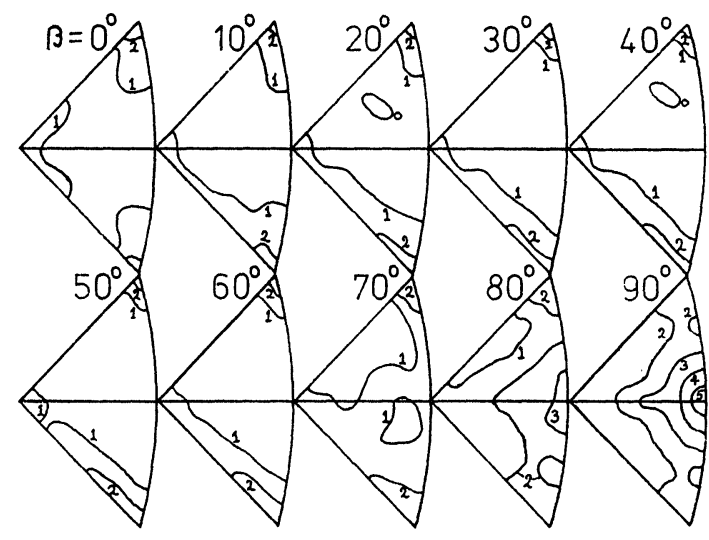

FIGURE 6 Biaxial pole figure for silicon iron rolled $60 \%$ and annealed at $700^{\circ} \mathrm{C}$.

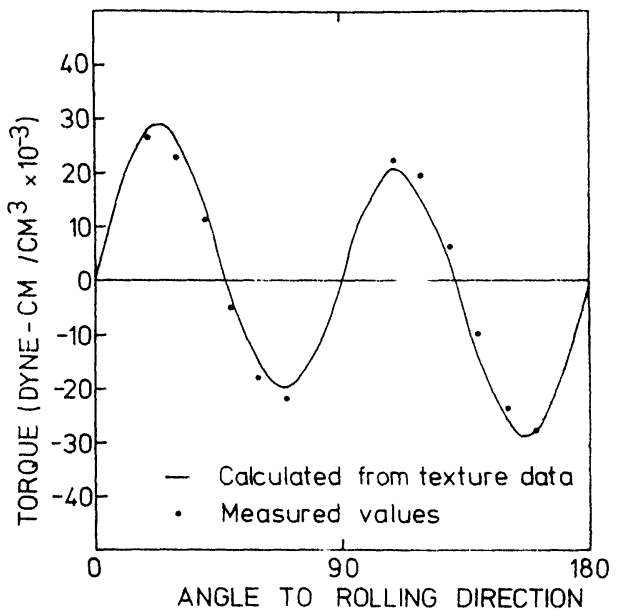

FIGURE 7 Torque magnetometer curve for rimming steel rolled $50 \%$ and annealed at $700^{\circ} \mathrm{C}$.

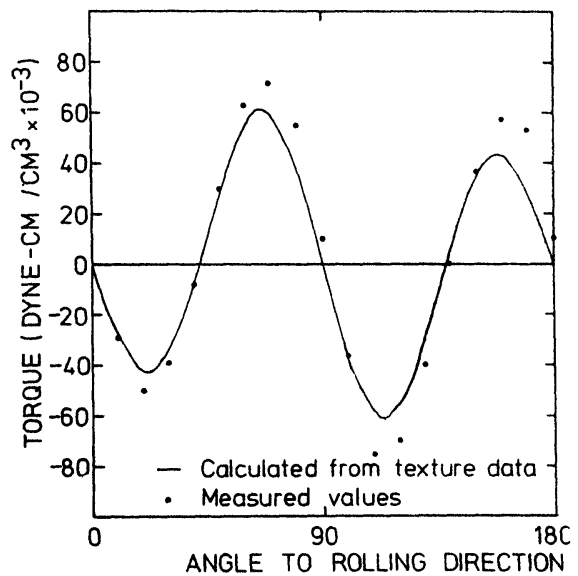

FIGURE 8 Torque magnetometer curve for rimming steel rolled $90 \%$. 
where. ${ }^{15}$ Very close agreement is obtained in most cases, confirming the applicability of the method and the texture analysis. The $90 \%$ rolled specimen gives qualitative agreement, but quantitatively the results are not so good as in the other cases. It seems possible that here the texture is too sharp to be completely explained by the analysis used. Indeed, the discrepancies between measured and regenerated pole figures were noticeably larger than for the other specimens. At the lower reductions ${ }^{15}$ the degree of fit is equally good for the cold worked and the recrystallised samples, and it is clear that the magnetocrystalline anisotropy coefficients are not significantly affected by deformation.

In the analysis of power loss and a number of other magnetic properties one requires to distinguish between contributions from crystal orientation, and those which derive from the microstructure. For cubic crystal symmetry, any directional property is capable of expression in series form as in Eq. (1). Provided that there are no interactions between grains, the value for a polycrystal may be obtained by averaging the single crystal values weighted by the orientation density of each. One can thus express a general property $P$ as:

$$
\begin{aligned}
P=A_{0}+A_{1}\left[\left(l^{2} m^{2}+\right.\right. & \left.\left.m^{2} n^{2}+n^{2} l^{2}\right) t\right]_{\mathrm{Av}} \\
& +A_{2}\left[\left(l^{2} m^{2} n^{2}\right) t\right]_{\mathrm{Av}}+\ldots
\end{aligned}
$$

where the average is carried out over a sufficiently large volume of orientation space. This approach has been outlined by Bunge ${ }^{13}$ in relation to texture description using spherical functions. In the present work it was found that the bracketed terms for $A_{2}$ and higher orders were much smaller than that for $A_{1}(<5 \%)$ and could reasonably be neglected. The physical property in question could then be described by the two coefficients $A_{0}$ and $A_{1}$, the former representing the orientation-independent part of the property and the latter representing its orientation-dependent part. Using direct measurements of the property in question along seven directions around the sheet and values of $\left[\left(l^{2} m^{2}+m^{2} n^{2}+n^{2} l^{2}\right) t\right]_{A v}$ calculated for the same directions from the orientation distribution, the values of $A_{0}$ and $A_{1}$ were obtained using a least squares solution.

Figure 9 shows the measured values of total power loss at three frequencies and the curves fitted to these using the texture data. It is seen that both coefficients $A_{0}$ and $A_{1}$ increase with frequency. $A_{1}$ varies almost linearly while $A_{0}$ shows a slightly stronger frequency dependence. The proportion of

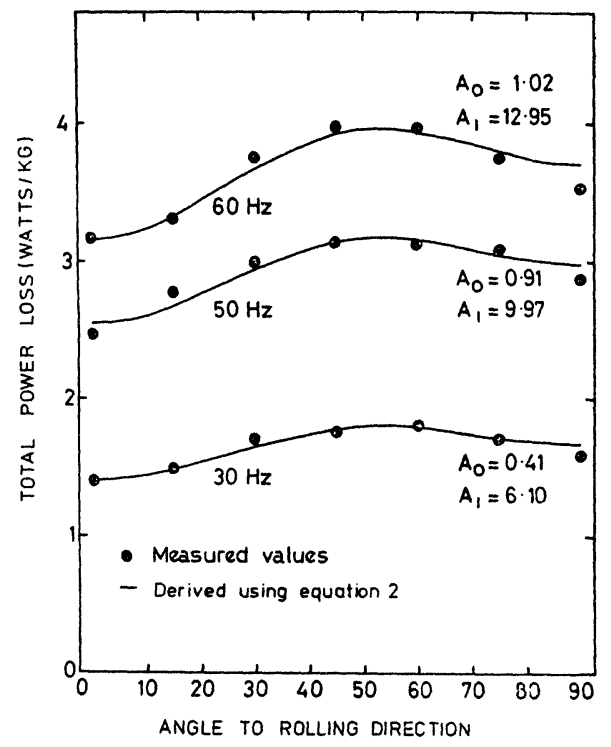

FIGURE 9 Total power loss data for the silicon iron.

the total power loss which is independent of texture is approximately $25 \%$ in this material. For ideally oriented sheets with the same microstructure and dimensions, the total loss would be simply equal to $A_{0}$ since the direction cosines expression would reduce to zero. This can thus provide a target at which to aim when developing textured steels for magnetic applications. Another application of the procedure described here is in the calculation of physical properties along directions where direct measurement is not possible. Knowledge of the orientation distribution and the coefficients $A_{0}$ and $A_{1}$ allows determination of the property along any direction in the polycrystalline aggregate.

In Figure 10, plots are presented of measured and derived values obtained from the static magnetisation curve. In the case of the hysteresis loss (B-H loop area), a small negative value of $A_{0}$ was found using the least squares analysis. Since this is physically unreasonable, the value of $A_{0}$ was set to zero which caused only a very small change in the $A_{1}$ term. Multiplying this loss/cycle value by the frequency gives the contribution of hysteresis to total power loss. Table I summarises these results and also the calculated classical eddy current losses.

Two important conclusions may be drawn from the data of Table I. Since the $A_{1}$ coefficients for total loss and hysteresis loss are the same within experimental error, then the orientation dependence of power loss is entirely due to the hysteresis contribution. Secondly, the classical eddy current loss 


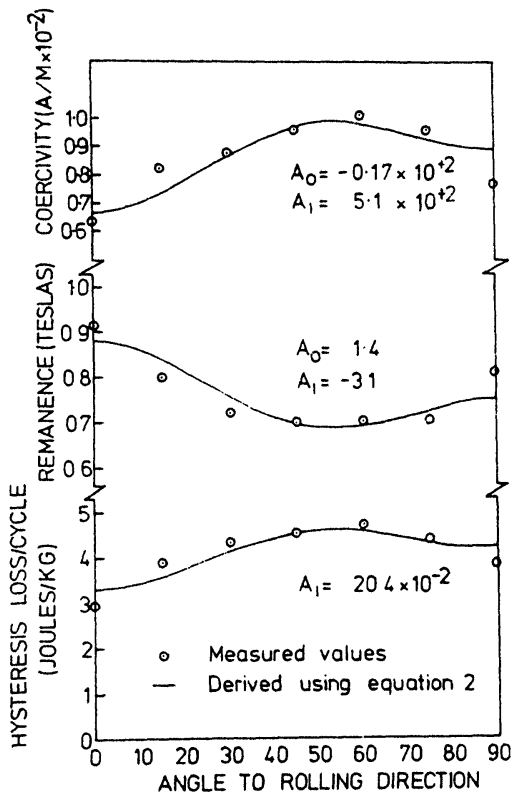

FIGURE 10 Permeameter results for the silicon iron.

is smaller than the term $A_{0}$ of the total power loss, indicating that there is an additional orientationindependent part of the power loss present. This additional term is of similar magnitude to that calculated from classical eddy current theory and is approximately proportional to frequency. It is possible that this loss derives from micro eddy current at moving domain boundaries, which was the mechanism suggested by Pry and $\mathrm{Bean}^{3}$ to explain a similar magnetic anomaly.

\section{TABLE I}

Loss coefficients and calculated eddy current losses in units of watts $/ \mathrm{kg}$

\begin{tabular}{cccccc}
\hline Frequency & $\begin{array}{c}\text { Total } \\
\text { Power Loss } \\
\text { Coefficients }\end{array}$ & $\begin{array}{c}\text { Hysteresis } \\
\text { Loss } \\
\text { Coefficients }\end{array}$ & $\begin{array}{c}\text { Classical } \\
\text { Eddy Current } \\
\text { Loss }\end{array}$ \\
\hline & $\mathrm{A}_{0}$ & $\mathrm{~A}_{1}$ & $\mathrm{~A}_{0}$ & $\mathrm{~A}_{1}$ & \\
$30 \mathrm{~Hz}$ & 0.41 & 6.10 & 0 & 6.1 & 0.14 \\
$50 \mathrm{~Hz}$ & 0.91 & 9.97 & 0 & 10.2 & 0.37 \\
$60 \mathrm{~Hz}$ & 1.02 & 12.95 & 0 & 12.2 & 0.54 \\
\hline
\end{tabular}

In Figure 10, attempts were also made to rationalise the orientation dependence of the remanence and the coercive force. The measured values are not very exact because of the rather small mass of specimen employed $(\sim 60 \mathrm{~g})$. Indeed, the fit here is not good, although the correct trends are present, and for coercive force the coefficient $A_{0}$ is negative which is physically unreasonable. There is another possible source for the discrepancy between actual behaviour and theoretical prediction. For an exact application of Eq. (2), each grain should be subject to identical conditions e.g. constant, field or constant induction. In a typical polycrystal, however, the magnetic flux may be locally short-circuited along easy paths and avoid unfavourably oriented grains. Such behaviour is hard to quantify and its importance probably depends on the particular property under consideration.

\section{CONCLUSIONS}

Good agreement is obtained between measured torque magnetometer data and computed values based on orientation distributions derived from $\mathrm{x}$-ray diffraction measurements.

Using orientation distribution data it is possible to analyse measured magnetic property data into orientation-dependent and orientation-independent components. For a.c. power loss values, the former contribution derives entirely from the normal hysteretic component. The classical eddy current loss and an anomalous loss of approximately the same value constitute the orientation-independent component. The analysis employed is quite general and may be used to obtain values of the desired physical property for a range of conditions.

\section{ACKNOWLEDGMENTS}

The authors are most grateful to Dr. R. O. Williams for making available the "Biaxial" computer program. They also thank the British Steel Corporation, Strip Mills Division, for financial assistance and for help with the testing of magnetic properties.

\section{REFERENCES}

1. P. N. Goss, Trans. A.S.M. 23, 515, (1935).

2. R. M. Bozorth, Ferromagnetism (D. Van Nostrand, 1951), p. 778.

3. R. H. Pry and C. P. Bean, J. Appl. Phys. 29 (3), 532, (1958).

4. E. W. Lee, Proc. I.E.E. 105C, 337, (1958).

5. K. J. Overshott, I. Preece and J. E. Thompson, Proc. I.E.E. 115, 1840 (1968).

6. M. McCarty, G. L. Houze and F. A. Malagari, J. Appl. Phys. 38, 1096 (1967).

7. M. R. Daniels, I.E.E. Conf. Publ. 33 (1967), pp. 4-8.

8. N. S. Akulov, Z. Phys. 54, 582 (1929).

9. L. P. Tarasov and F. Bitter, Phys. Rev. 52, 353 (1937).

10. R. O. Williams, Trans. TMS-AIME 242, 105 (1968).

11. H. J. Bunge, $Z$. Metallkunde 56, 872, (1965).

12. R.-J. Roe, J. Appl.Phys. 36, 2024, (1965).

13. H. J. Bunge, Mber. dtsch. Akad. Wiss. 3, 97 (1961). 
14. H. J. Bunge, Phys. Stat. Sol. 26, 167 (1968).

15. W. B. Hutchinson and J. G. Swift, to be published in J. Pol. Acad. Sci.

16. A. J. Heckler and W. G. Granzow, Met. Trans. 1, 2089 (1970).

17. L. K. Jetter and B. S. Borie, J. Appl. Phys. 24, 532,(1953).
18. I. L. Dillamore, C. J. E. Smith and T. W. Watson, Met. Sci.J., 1, 49 (1967).

19. I. L. Dillamore, P. L. Morris, C. J. E. Smith and W. B. Hutchinson, to be published in Proc. Roy. Soc.

20. P. L. Morris and W. B. Hutchinson, to be published.

21. Ref. 2, p. 567. 